\title{
Accuracy of a Navigation System for Computer-Aided Oral Implantology
}

\author{
Wolfgang Birkfellner ${ }^{1 \star}$, Felix Wanschitz ${ }^{2}$, Franz Watzinger ${ }^{2}$, Klaus Huber ${ }^{1}$, \\ Michael Figl ${ }^{1}$, Christian Schopper ${ }^{2}$, Rudolf Hanel ${ }^{3}$, Franz Kainberger ${ }^{3}$, \\ Joachim Kettenbach ${ }^{4}$, Sanda Patruta ${ }^{5}$, Rolf Ewers ${ }^{2}$, and Helmar Bergmann ${ }^{1,6}$ \\ 1 Department of Biomedical Engineering and Physics \\ 2 Department of Oral and Maxillofacial Surgery \\ 3 Department of Diagnostic Radiology, Division of Osteoradiology \\ 4 Department of Diagnostic Radiology, \\ Division of Angiography and Interventional Radiology \\ ${ }^{5}$ Dental School, University of Vienna \\ ${ }^{6}$ Ludwig-Boltzmann Institute of Nuclear Medicine \\ General Hospital, University of Vienna, \\ Waehringer Guertel 18-20, A-1090 Vienna, Austria
}

\begin{abstract}
Placement of endosteal implants is a widespread therapy for re-establishing full functionality in edentulous patients. As a first application of VISIT, a modular software system for research into computeraided surgery developed at our hospital, we have implemented a navigation system for computer-aided implant dentistry (CAID). Besides improved accuracy, benefits of CAID include fast translation of preoperative imaging to the operating theatre and the possibility to insert the implants without having to prepare large mucosa flaps. In this cadaver study, we have measured the overall accuracy of VISIT for inserting four intraforaminal implants in the edentulous mandible. Five cadaver mandibles were embedded into plaster. After high-resolution CT scanning, the mandibles were registered, and the implant channels were drilled by the surgeon. Training implants were inserted into the implant channels, and the plaster was removed. Again, the mandibles underwent CT scanning, and the pre- and postoperative scans were registered relative to each other. A gross registration between pre- and postoperative scans was achieved using surface- or mutual information matching since in some cases the fiducial markers were lost. After transformation to a common coordinate system, the accuracy was assessed by measuring the distance of the implant's center to the cortex of the jawbone. Average accuracy of the navigation system was found to be $0.9 \pm 0.7 \mathrm{~mm}$, range $\{0.0 \ldots 3.5\} \mathrm{mm}$. We conclude that these results show that CAID is an interesting novel application of computer-aided surgery superior to conventional methods in oral surgery.
\end{abstract}

\footnotetext{
^ e-mail: Wolfgang.Birkfellner@univie.ac.at
} 


\section{Introduction}

Computer-aided insertion of endosteal implants is a rather novel application of computer-aided surgery (CAS). Basically, small titanium implants are placed in the edentulous jaw [1277.9. In this cadaver study, we have assessed the precision of VISIT's module for computer-aided implant dentistry (CAID). VISIT is a modular software system for fast development of exploratory research applications in CAS developed at our hospital.

High-resolution computed tomography (CT) provides the imaging modality for preoperative planning of implant channels. This is a widespread technique in oral surgery; usually, the preoperative plan is transferred to the operating theatre by means of templates [5]. This requires an additional step for manufacturing the template after planning. Furthermore, some template techniques do also require preparation of large mucosa flaps for inserting the template in the patient's oral cavity. This is undesirable since the atrophic jaw is to a large extent vascularized by the periost over the mucosa tissue, and temporary removal of the mucosal tissue thus increases atrophy of the available bone volume.

Another interesting application of CAID is the insertion of endosteal implants in the zygoma; this allows for using endosteal implants for coverage of large skeletal defects after hemimaxillectomy in tumour patients 8 . In this case, CAS allows for accessing the zygoma for drilling an implant channel from a minimalinvasive external approach; this is not feasible in normal surgery due to the vicinity of critical anatomical structures such as the eye. In this study, we have assessed the accuracy achievable in implant placement, extending a first cadaver study on cadaver skulls for zygoma implants [410].

\section{Materials and Methods}

\subsection{Hard- and Software}

VISIT, our experimental navigation system development platform, consists of an interface for communication with an optical tracking system (Flashpoint 5000, Image Guided Technologies, Boulder/CO) written in ANSI-C using the POSIX.1 standard routines for serial communication, and a platform-independent ANSI$\mathrm{C}$ program. Image processing was added using AVW (Biomedical Imaging Resource, Mayo Clinic, Rochester/MN) [3]. The graphical user interface was programmed using Tcl/Tk 8.02. Currently, the program is implemented on a SGI O2 RS12000 workstation running IRIX 6.5.6. Sterilizable LED probes for tracking the patient and the surgical drill were also developed at our hospital [1.

\subsection{Experimental Protocol}

Three miniature osteosynthesis screws (Leibinger AG, Freiburg/Germany) were implanted into five cadaver mandibles preoperatively. The mandibles underwent 


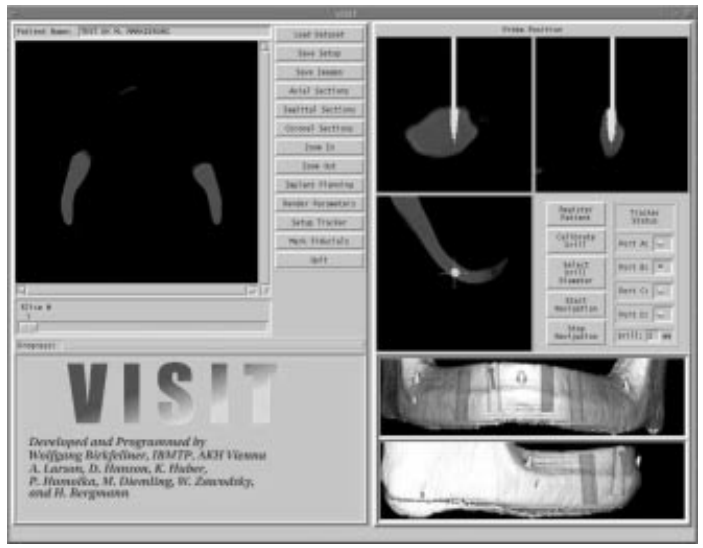

Fig. 1. Main screen of VISIT's CAID module. The position of the surgical drill is visualized using oblique reformatting and projection over volume renderings.

high resolution CT scanning (conventional CT, $0.25 * 0.25 * 1.00 \mathrm{~mm}^{3}$ ). After scanning, the mandibles were embedded into plaster so that only the remaining alveolar ridge remained visible. Registration of mandible and CT-scan was performed using a point-to-point registration algorithm [6]. Specialized tools developed for CAID [1,2,3] consisting of a dynamic reference frame (DRF) for tracking the patient and a probe attached to a surgical drill were used. Four intraforaminal implants were planned on the CT-scans. After drilling the implant channels, four IMZ training implants (Friatec AG, Mannheim/Germany) with 4 $\mathrm{mm}$ diameter and $13 \mathrm{~mm}$ length were inserted.

\subsection{Postoperative Assessment}

After implant insertion, the plastercast was removed and the mandibles underwent CT scanning again. Both the pre- and postoperative scans were interpolated to isotropic voxel size; since in some cases the fiducial markers were lost, the preand postoperative scans were matched either by using mutual information or by surface registration. After computing the registration, the postoperative scan was transformed to the coordinate system of the preoperative scan. The position of both the implant tip and the implant's head were then measured on both scans using axially reformatted slices. The deviation was defined as the difference between the distances between implant tip and base to the lingual and buccal cortex of the jawbone. Furthermore, the distance of the implant's center to a fiducial marker placed on the mentum was measured. All image processing besides

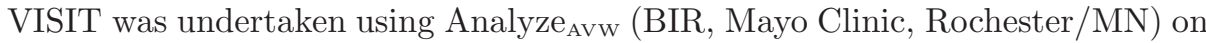
a SGI O2 RS12000 and Analyze PC on a Intel-based PC running Windows NT 4.0 . 


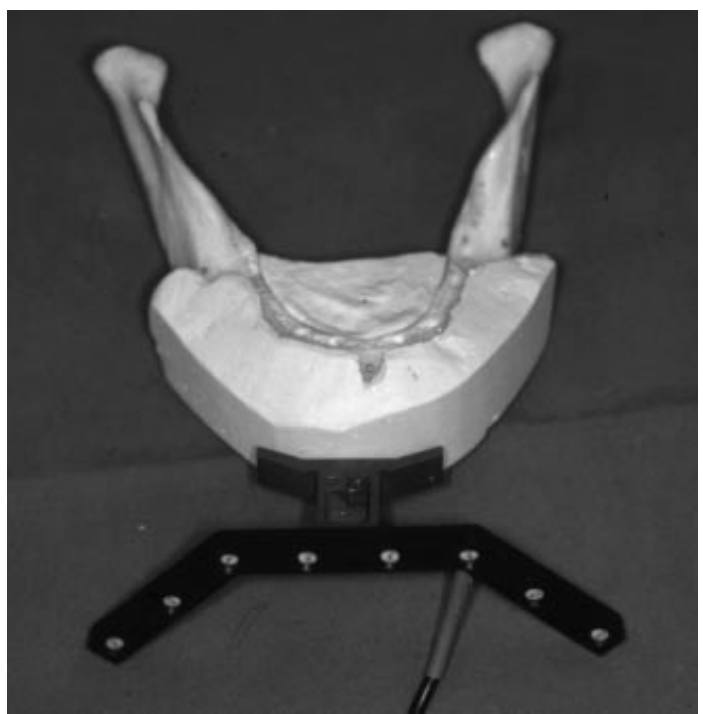

Fig. 2. A cadaver mandible. Visible is the plastercast surrounding the mandible during the surgical procedure, and the optical probe for tracking the position of the mandible during surgery.

Table 1. Average deviation between implant positions on pre- and postoperative scans as measured on five cadaver mandibles. As a measure of accuracy, the distance between the lingual and buccal cortex of the jawbone and the implant's tip (embedded in the mentum) and base (on the alveolar ridge) was measured. Furthermore, the deviation between central axis of the implant and a fiducial markers on the mentum was measured on pre- and postoperative scans and compared. All measurements in mm.

\begin{tabular}{|c|c|c|c|c|}
\hline$\Delta \operatorname{Tip}_{\text {lingual }}$ & $\Delta \operatorname{Tip}_{\text {buccal }}$ & $\Delta$ Base $_{\text {lingual }}$ & $\Delta$ Base $_{\text {buccal }}$ & $\Delta$ Axis-Fiducial \\
\hline $1.4 \pm 0.7$ & $1.4 \pm 0.8$ & $0.5 \pm 0.4$ & $0.5 \pm 0.3$ & $0.9 \pm 0.7$ \\
\hline
\end{tabular}

\section{Results}

Average deviation from the preoperatively planned position was found to be 0.9 $\pm 0.7 \mathrm{~mm}$. No perforation of the cortices did occur. Furthermore, the foramen mentale, the exit point of the Nervus alveolaris inferior, was not penetrated; therefore the nerve would not have been anaesthesized in a live patient despite the fact that the foramen mentale was not visible during the experiments and two of the implants were planned close to the foramina in each specimen. Additional time expense due to the navigation procedure was maintained within a few minutes as compared to the conventional procedure.

\section{Discussion}

There is an obvious need for transferring the preoperative plan of the implant's position to the operating theatre. This is especially the case in completely edentulous patients with highly atrophic jawbone. Typically, these patients have to undergo augmentative surgery (for instance by implanting small chips of pelvic 


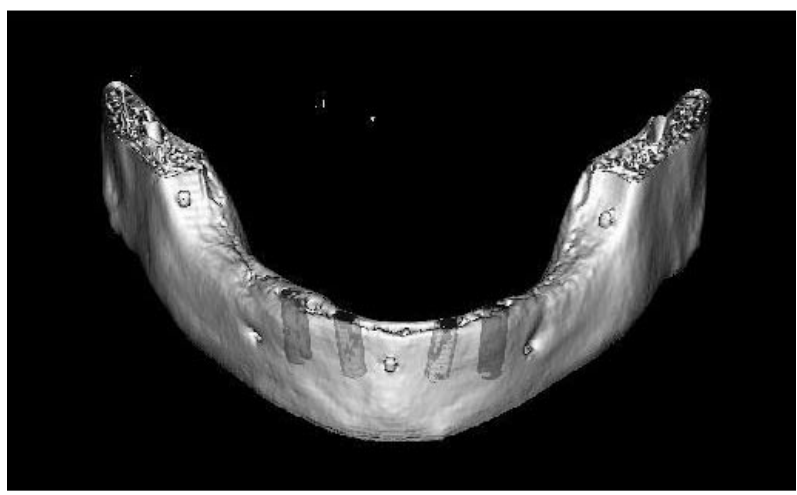

Fig. 3. Comparative view of planned (upper image) and postoperative implant position (lower image). Images were made after reg-

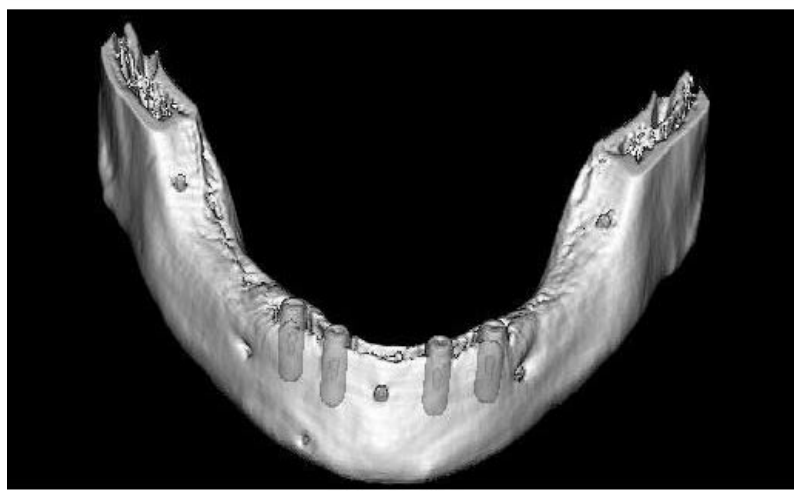
istration of the pre- and postoperative scan. In this case, average deviation of the planned and true implant position was found to be $0.7 \mathrm{~mm}$.

bone under the mucosa of the maxillary sinus) prior to implantation, thus presenting an unphysiological anatomic situation. Preoperative CT-scanning and implant planning on the three-dimensional CT scan is an accepted therapy to make optimum implant placement sure in these cases. Typically, a template is produced to convey the preoperative plan to the operating theatre. The proposed navigation method is superior to this technique due to two reasons. First of all, the template has to be made by a dental technician, which is usually a time-consuming, cost-intensive process. Second, the template has to rest on a repeatable position in the patient's oral cavity; this can be achieved by preparing a large mucosa flap which exhibits most of the jaw's cortex. This technique is typical in implant dentistry since the flap also provides visual control for not penetrating the cortex. The mucosal flap, however, is not desirable since the jawbone's blood supply mostly stems from the periost. As said before, further atrophy of the jawbone is likely to occur due to this method.

Compared to an earlier series of cadaver studies [4,10, we were able to improve the performance of VISIT by using newly developed LED assemblies. Taking into account the remaining mechanical problems in the surgical drill (namely the loose fit of the drill bit in the surgical drill), we conclude that accuracy achievable with the navigation system is sufficient for this type of application, especially since the experiments presented in this paper show a real-life situation; 


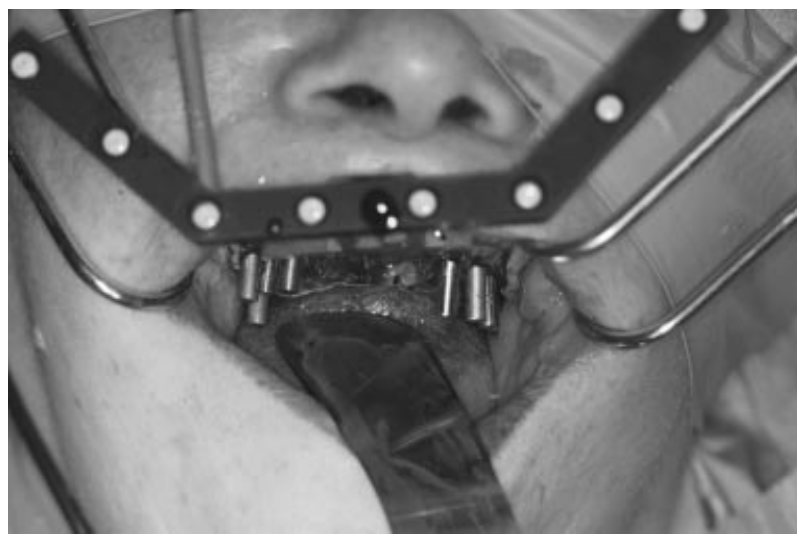

Fig. 4. Intraoperative view during the first operation with VISIT. Eight implants were placed in a completely edentulous, atrophic maxilla. Prior to the operation, the patient underwent augmentative surgery to increase the amount of bone volume available for implantation. Visible are six parallelization nails and the dynamic reference frame for tracking the maxilla.

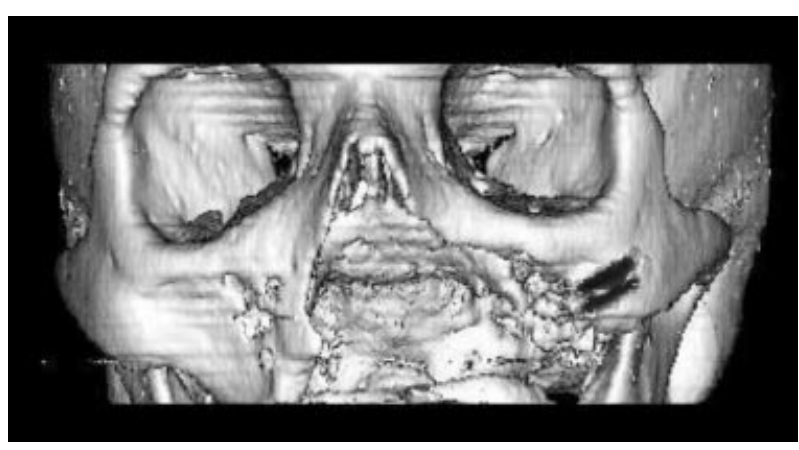

Fig. 5. Postoperative CT scan of a patient who underwent insertion of two endosteal implants in the zygoma; the maxilla and the hard palate were removed in an earlier operation due to squamous cell carcinoma of the palate. The implants were inserted for fixation of an obturator prosthesis which seals the nasopharynx.

the implant channels were drilled by a surgeon, and additional problems such as the deviation of the planned path due to hard trabecular bone or increasing deviation in the course of widening the $2 \mathrm{~mm}$ pilot hole to the full diameter of $4 \mathrm{~mm}$ ocurred. The problem of deviations due to the loose fit of the drill bit and flexion of the drill is also documented by the fact that the accuracy at the implant tip is worse than in the case of the implant base.

The reliability of the system was found to be sufficient for first intraoperative use in December 1999 (Fig. 4) in an edentulous patient. Since then, two more patients which underwent hemimaxillectomy due to squamous cell carcinoma or adenocystic caricinoma were operated. Fig 4 shows a patient after insertion of two $4 \times 15 \mathrm{~mm}$ Friatec IMZ implants into the zygoma for placing an obturator prosthesis 410; this prosthesis seals the nasopharynx after loss of the hard palate. 


\section{Acknowledgment}

We wish to thank W. Piller (L.-Boltzmann Institute of Nuclear Medicine, Vienna), S. Baumgartner, A. Taubeck, and A. Gamperl (Dept. of Biomedical Engineering and Physics, Vienna). The implants were provided by Schütze Dentaltechnik, Vienna. A. Larson, D. Hanson and the staff at BIR (Mayo Clinic, Rochester/MN) were of great help during the development of VISIT. AVW, the library used for VISIT's image processing capabilities, was provided courtesy of Dr. R. A. Robb. This work was supported by the Austrian Science Foundation FWF under research grant P12464-MED.

\section{References}

1. W. Birkfellner, P. Solar, A. Gahleitner et al.: "Computer - Aided Implant Dentistry - An Early Report", in A. Colchester, C. Taylor (eds.): "Medical Image Computing and Computer Aided Interventions - MICCAI'99", Springer LNCS 1679, 883-891, (1999).

2. W. Birkfellner, P. Solar, A. Gahleitner et al.: "In-vitro assessment of a registration protocol for image guided implant dentistry", Clin Oral Impl Res, in press.

3. W. Birkfellner, K. Huber, A. Larson et al.: "A modular software system for computer aided surgery and it's first application in oral implantology", IEEE Trans Med Imaging, in press.

4. W. Birkfellner, F. Watzinger, F. Wanschitz et al.: "Image Guided Insertion of Endosteal Implants in the Zygoma for Reconstructive Purposes - A Pilot Study", to appear in Proceedings of SPIE Medical Imaging 2000.

5. G. Champleboux, T. Fortin, H. Buatois et al.: "A fast, accurate and easy method to position oral implants using computed tomography", in W. M. Wells, A. Colchester, S. Delp (eds.): "Medical Image Computing and Computer-Aided Interventions MICCAI'98", Springer LNCS 1496, 269-276, (1998).

6. B. K. P. Horn, "Closed form solution of absolute orientation using unit quaternions", J Opt Soc Am A 4(4), 629 - 642, (1987).

7. O. Ploder, A. Wagner, G. Enislidis et al.: "Computer-assisted intraoperative visualization of dental implants. Augmented reality in medicine", Radiologe 35(9), 569-572, (1995).

8. E. D. Roumanas, R. D. Nishimura, B. K. Davies et al.: "Clinical evaluation of of implants retaining edentulous maxillary obturator prostheses", J Prosthet Dent 77(2), 184 pp., (1997).

9. F. Watzinger, W. Birkfellner, F. Wanschitz et al.: "Positioning of dental implants using computer-aided navigation and an optical tracking system: case report and presentation of a new method", J Craniomaxfac Surg 27, 77-81, (1999).

10. F. Watzinger, W. Birkfellner, F. Wanschitz et al.: "Placement of endosteal implants in the zygoma after maxillectomy: a cadaver study using surgical navigation", Plast Reconstr Surg, in press. 